\title{
Comparison of serum human epididymis protein 4 and carbohydrate antigen 125 as markers in ovarian cancer: A meta-analysis
}

\author{
SHUAI ZHEN ${ }^{1,2}$, LI-HONG BIAN ${ }^{3}$, LI-LI CHANG ${ }^{2}$ and $\mathrm{XIN} \mathrm{GAO}^{2}$ \\ ${ }^{1}$ Xijing Hospital, Fourth Military Medical University, Xi'an, Shaanxi 710032; ${ }^{2}$ Department of Pharmacology and Toxicology, \\ Beijing Institute of Radiation Medicine, Beijing 100850; ${ }^{3}$ Department of Gynecology, \\ Affiliated Hospital of Academy of Military Medical Sciences, Beijing 100850, P.R. China
}

Received January 15, 2014; Accepted March 26, 2014

DOI: $10.3892 / \operatorname{mco} .2014 .279$

\begin{abstract}
Ovarian cancer (OC) is the third most common type of gynecological cancer. Measurements of human epididymis protein 4 (HE4) levels have been suggested for improving the specificity of the laboratory identification of OC. For this meta-analysis, the Medline, Embase and Cochrane databases were searched to identify relevant studies. All the included studies for diagnostic performance were combined with sensitivity, specificity, positive likelihood ratio, negative likelihood ratio, diagnostic odds ratios (DORs) with $95 \%$ confidence intervals (CIs), summary receiver operating characteristic (SROC) curves and areas under the SROC curves (AUC). A total of 25 studies including 4,729 patients were identified as eligible for inclusion in the final analysis. The pooled sensitivities and respective $95 \%$ CIs for HE4 and carbohydrate antigen 125 (CA125) were 0.74 (0.72-0.76) and 0.74 (0.72-0.76), respectively. The pooled specificities and respective $95 \%$ CIs for HE4 and CA125 were 0.90 (0.89-0.91) and 0.83 (0.81-0.84), respectively. The summary DORs and 95\% CIs for HE4 and CA125 were 43.35 (29.13-64.51) and 17.06 (10.97-26.51), respectively and the AUCs for HE4 and CA125 were 0.8915 and 0.8538 , respectively. In total, 9 studies investigated the diagnostic accuracy of HE4 combined with CA125 for the diagnosis of OC. The pooled sensitivity and 95\% CIs of HE4, CA125 and HE4+CA125 in this subgroup were 0.71 (0.67-0.75), 0.74 (0.69-0.78) and 0.90 (0.87-0.92), respectively; the pooled specificity and 95\% CIs of HE4, CA125 and HE4+CA125 were 0.92 (0.90-0.94), 0.73 (0.69-0.76) and 0.85 (0.82-0.87), respectively. The diagnostic accuracy of HE4 in distinguishing $\mathrm{OC}$ from other benign gynecological diseases was found to be
\end{abstract}

Correspondence to: Professor Xin Gao, Department of Pharmacology and Toxicology, Beijing Institute of Radiation Medicine, 27 Taiping Road, Beijing 100850, P.R. China

E-mail: gaox_amms@126.com

Key words: ovarian cancer, human epididymis protein 4, carbohydrate antigen 125 , meta-analysis to be superior to that of CA125 and the combination of HE4 and CA125 may enhance the diagnostic sensitivity.

\section{Introduction}

Ovarian cancer (OC) is the third most common tumor of the female genital tract after carcinomas of the cervix and endometrium and remains the leading cause of gynecological malignancy-related mortality (1). In total, $75 \%$ of the patients are diagnosed at an advanced stage and the 5-year survival rate is consequentially poor, since OC is generally asymptomatic in its early stages and there is currently no effective screening method (2). Advances in the resolution of sonography have increased its accuracy for the differential diagnosis of OC; however, the results may vary with differences in equipment and among different operators. With the development of genomics and proteome analysis, an increasing number of tumor markers have been introduced to aid the diagnosis of cancer and have become an important and convenient diagnostic tool. It is difficult to detect OC at its early stages using conventional methods. Therefore, there is a need for biomarkers of higher diagnostic accuracy to distinguish malignant from benign pelvic masses at an early stage and set up an effective screening program (3).

Carbohydrate antigen 125 (CA125) measurement is currently considered to be a significant component in the workup of a patient with an adnexal mass and is the standard biomarker for detecting OC recurrence and monitoring treatment efficacy. However, the application of CA125 is compromised by its low specificity, particularly in premenopausal women, as the CA125 levels may be elevated above normal in a number of common benign gynecological conditions and in other malignancies (4). Therefore, considerable efforts are aimed at identifying novel markers, which are more sensitive and specific compared to CA125 and may be used in combination with or instead of CA125 to improve the diagnosis of OC $(5,6)$.

Among a wide spectrum of biomarkers, including CA125, TPA and TAG72, human epididymis protein 4 (HE4) has been proposed as a novel tumor marker for OC. Despite the low number of available studies, HE4 was reported to be used as an aid in the diagnosis of $\mathrm{OC}$, as it was found to be overexpressed in ovarian carcinomas but not in ovarian benign 
diseases, normal ovarian tissue or low-malignant potential tumors $(7,8)$. However, previous studies on the roles of HE4 and CA125 in the differential diagnosis of OC reported controversial and inconclusive results. Certain published studies and meta-analyses indicated that HE4 is not superior to CA125 in predicting OC $(9,10)$. Furthermore, it has not been determined whether diagnostic performance may be improved by combining measurements of HE4 and CA125, instead of each marker used alone. Therefore, we conducted a meta-analysis of the available evidence on the diagnostic accuracy of HE4 and CA125 by a stepwise selection of relevant studies, considering only those studies that evaluated both markers in the same case series. These data may provide evidence supporting further application of HE4 in the diagnosis of OC.

\section{Materials and methods}

Data sources and search strategy. We followed the Meta-analysis Of Observational Studies in Epidemiology (12) and the Cochrane Handbook for Systematic Reviews of Diagnostic Test Accuracy and conducted this meta-analysis in accordance with the Preferred Reporting Items for Systematic Reviews and Meta-analyses guidelines (11). A prespecified protocol, including data sources, search strategy, inclusion/exclusion criteria for the articles and methods for analysis, was developed prior to the initiation of the study. A systematic review of original articles analyzing the diagnostic performance of HE4 and CA125 was performed by searching the Medline, Embase and Cochrane databases. Original and review articles published between 2008 and 2012 were sought. The search terms used were as follows: 'HE4/WAP 4-disulfide core domain 2 (WFDC2)', 'CA125', 'ovarian carcinoma/ovarian', 'sensitivity/specificity/false-negative/false-positive/diagnosis/detection/accuracy'. All the related publications were evaluated in order to retrieve the most eligible studies and their reference lists were searched manually to identify additional relevant publications. The aim of the search was to identify those articles in which HE4 and CA125 measurements were compared for OC diagnosis in order to provide a synthesis of evidence for the meta-analysis.

Inclusion/exclusion criteria. The authors evaluated the titles and abstracts of all the preliminary identified articles in order to assess whether the study was relevant to the aim of the meta-analysis. The complete study was evaluated using the following eligibility criteria for the meta-analysis of the studies: i) The sensitivity and specificity of HE4 and CA125 for the diagnosis of $\mathrm{OC}$ were provided; ii) the included patients were aged $\geq 50$ years; iii) the study design included patients with OC and evaluated the contribution of HE4 and CA125; iv) all the subjects were diagnosed by a gold standard (pathological examination of biopsied specimens), newly diagnosed patients with pathologically confirmed OC were the case group and patients with benign disease or healthy subjects were the control group; v) the diagnostic parameters were not of fixed specificity or sensitivity; vi) presence of data on sensitivity and specificity, or the possibility of deriving such values from the literature; vii) measurement of serum HE4 and CA125 in OC by ELISA or enzyme immunoassay with a clear cut-off value; and viii) the investigated population was represented by
Table I. QUADAS list.

\begin{tabular}{ll}
\hline Item no. & \multicolumn{1}{c}{ Description } \\
\hline 1 & Representative patient spectrum \\
2 & Clear description of selection criteria \\
3 & Acceptable reference standard \\
4 & Acceptable delay between tests \\
5 & Avoiding partial verification bias \\
6 & Sufficient differential verification bias \\
7 & Avoiding incorporation bias \\
8 & Sufficient description of index test \\
9 & Sufficient description of reference test \\
10 & Blinded interpretation of index test results \\
11 & Blinded interpretation of index reference results \\
12 & Availability of clinical data to the researchers \\
13 & Reporting of uninterpretable indeterminate results \\
14 & Explanation of withdrawals from study
\end{tabular}

QUADAS, quality assessment of diagnostic accuracy studies.

women with a gynecological disease suspected of being OC, which was the intended spectrum of patients to be investigated by circulating biomarker detection.

The exclusion criteria were as follows: i) Duplicate publications; ii) case reports; iii) insufficient data to construct a $2 \times 2$ table of the test results; iv) serum/plasma HE4 concentrations were measured to assess $\mathrm{OC}$ recurrence, monitor disease progression or treatment efficacy; v) lack of control group; and vi) abstracts, reviews, talks and review class documentations.

Data extraction and quality assessment. The data extracted from each study included name of first author, year of publication, country, number of patients, sensitivity, specificity, cut-off value, study design, patient selection and reconstructed $2 \times 2$ tables. Data were extracted from each study by two independent authors (Z.S. and B.L.H.). Any disagreements were resolved by consulting a third author (G.X.). The authors of the studies were contacted via e-mail in case of missing information.

The quality of the studies was assessed using a revised version of the Quality Assessment of Diagnostic Accuracy Studies (QUADAS-2) tool (13) (Table I) and the standards for reporting diagnostic accuracy tool (14). Each item scored 'yes', 'no' or 'unclear' if there was no sufficient information for an accurate judgment to be made.

Statistical analysis. We used the standard methods recommended for meta-analyses of diagnostic test evaluations (15). In this meta-analysis, the pooled sensitivity, pooled specificity, positive likelihood ratio (PLR), negative likelihood ratio (NLR), diagnostic odds ratio (DOR) and 95\% confidence interval $(\mathrm{CI})$ were calculated using the DerSimonian and Laird (16) method. In particular, the strength of the indication for the presence of the disease provided by the positive result of the test was relevant when PLR $>10$, moderate when $5<\mathrm{PLR}<10$ and poor when $2<\mathrm{PLR}<5$. The 


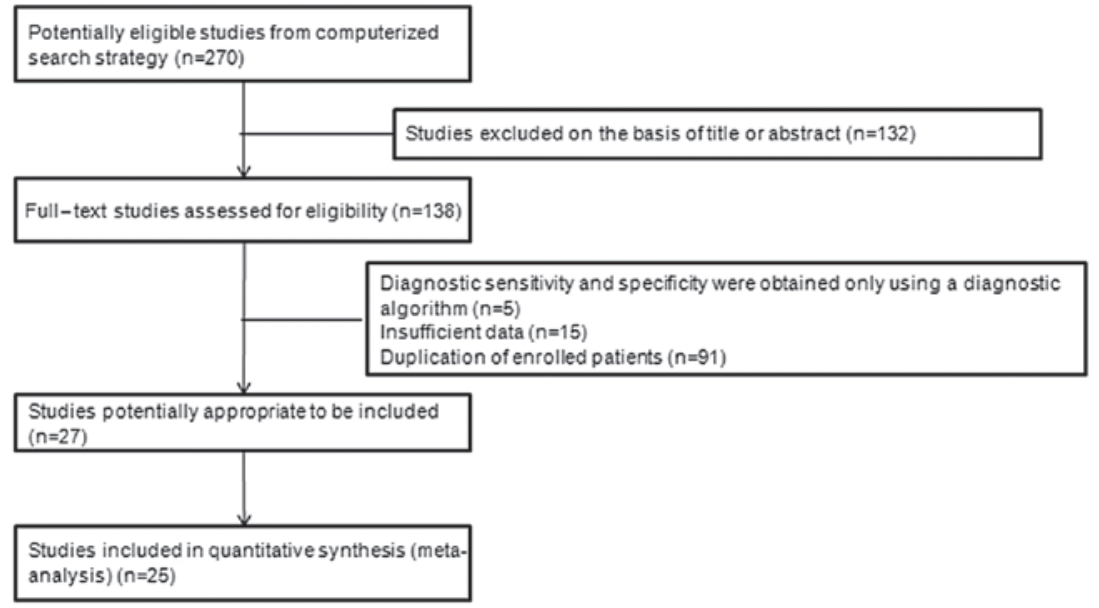

Figure 1. Flow diagram of the study selection process.
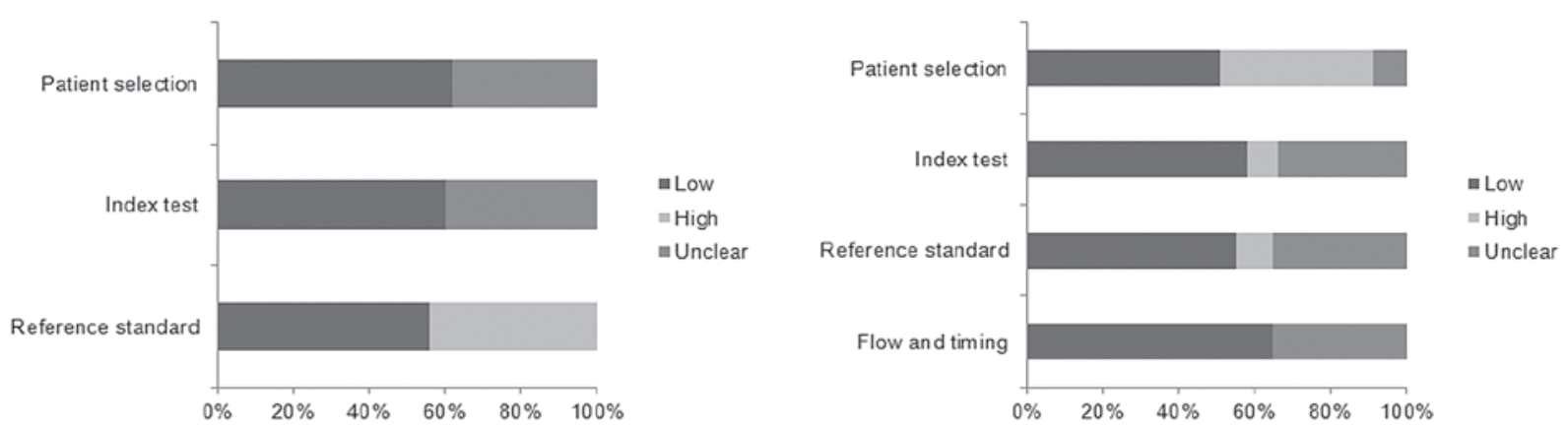

Figure 2. Graphical display of study characteristics according to the quality assessment of diagnostic accuracy studies-2 recommendations. Left, proportion of studies with low, high or unclear concerns regarding applicability; right, proportion of studies with low, high or unclear risk of bias.

strength of the indication for the absence of the disease provided by the negative result of the test was relevant when NLR $<0.10$, modest when $0.10<$ NLR $<0.20$ and poor when $0.20<\mathrm{NLR}<0.50$ (17). The DOR, as a single indicator measure of the accuracy of a diagnostic test (18), describes the odds of positive results in patients with the disease compared to the results in patients without disease. The present study used Moses' linear model to draw a summary receiver operating characteristic (SROC) curve, which summarized the joint distribution of sensitivity and specificity. The area under the SROC curve (AUC) was calculated and an AUC close to 1.0 signified that the test achieved almost perfect discrimination, while an AUC close to 0.5 indicated poor discrimination. The AUC was found to be useful to summarize the curve, but also quite robust to heterogeneity (19). Heterogeneity was assessed using the $\mathrm{LR} \mathrm{I}^{2}$ index and the $\chi^{2}$ test. The $\mathrm{I}^{2}$ index is a measure of the percentage of total variation across studies due to heterogeneity beyond chance; values $>50 \%$ indicate the presence of heterogeneity (20). A P-value of $<0.05$ calculated by the $\chi^{2}$ test was considered to indicate a statistically significant difference. In case of heterogeneity, the random effects model was used (21). All the analyses were performed using the Meta-Analysis of Diagnostic and Screening Test (Meta-DiSc) program, version 1.4 (Ramon y Cajal Hospital, Madrid, Spain) and Review Manager (RevMan) version 5 (The Nordic Cochrane Centre, The Cochrane Collaboration, Copenhagen, Denmark).

\section{Results}

Study characteristics. Based on the aforementioned search terms, a total of 270 articles were identified. After scanning the titles and abstracts, 132 articles were excluded. Subsequently, a further 111 articles were excluded for the following reasons: 5 only used a diagnostic algorithm, 15 presented insufficient data and 91 had duplicate patient enrollment. Finally, a total of 25 studies (2,22-45) with 4,729 patients fulfilled all the inclusion criteria and were considered for the analysis (Fig. 1). The target population of the study was women in a preoperative setting or with a known adnexal mass. The characteristics and results of the included studies are summarized in Table II. According to the QUADAS-2 checklist, details on selection, data collection and enrolment were retrieved. As shown in Table II, the enrolment differed widely among studies: Sample size and OC prevalence, setting of data collection, patient characteristics (prevalence of women of postmenopausal status) and severity of OC (prevalence of late stage-disease). Each of these points may represent a source of heterogeneity among studies.

Approximately $60 \%$ of the studies were performed in a gynecological oncology setting, which suggested a different assessment and a higher grade of severity for OC. The remaining studies, including early-stage OCs, were performed in a gynecological setting. The wide variation in the prevalence of women of postmenopausal status across the studies was likely to affect diagnostic performance. 
A

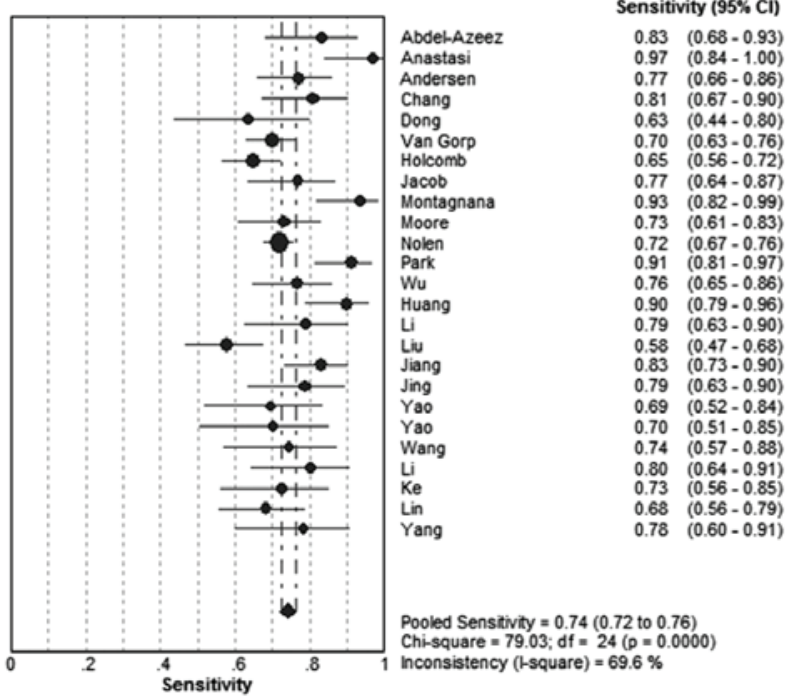

C

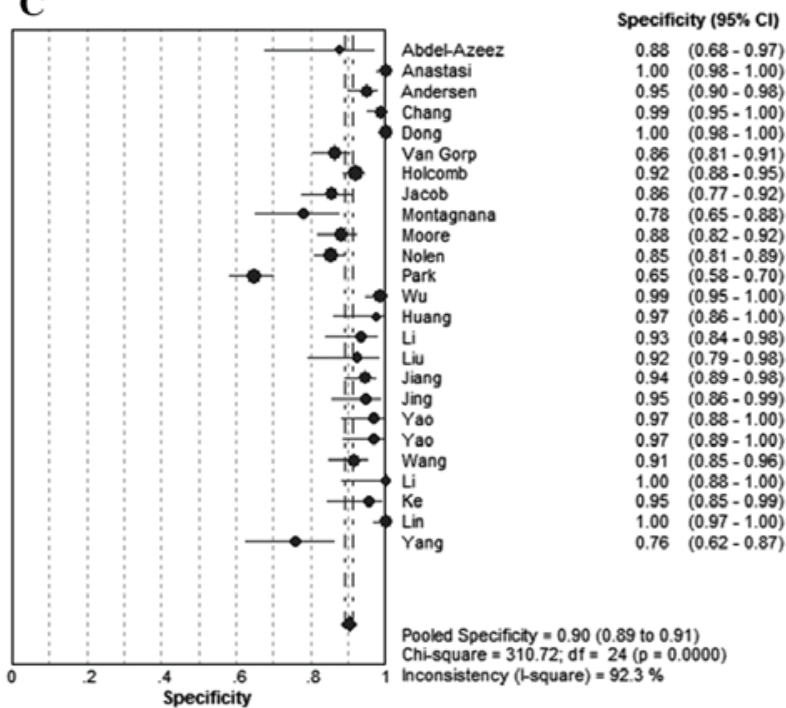

$\mathbf{E}$

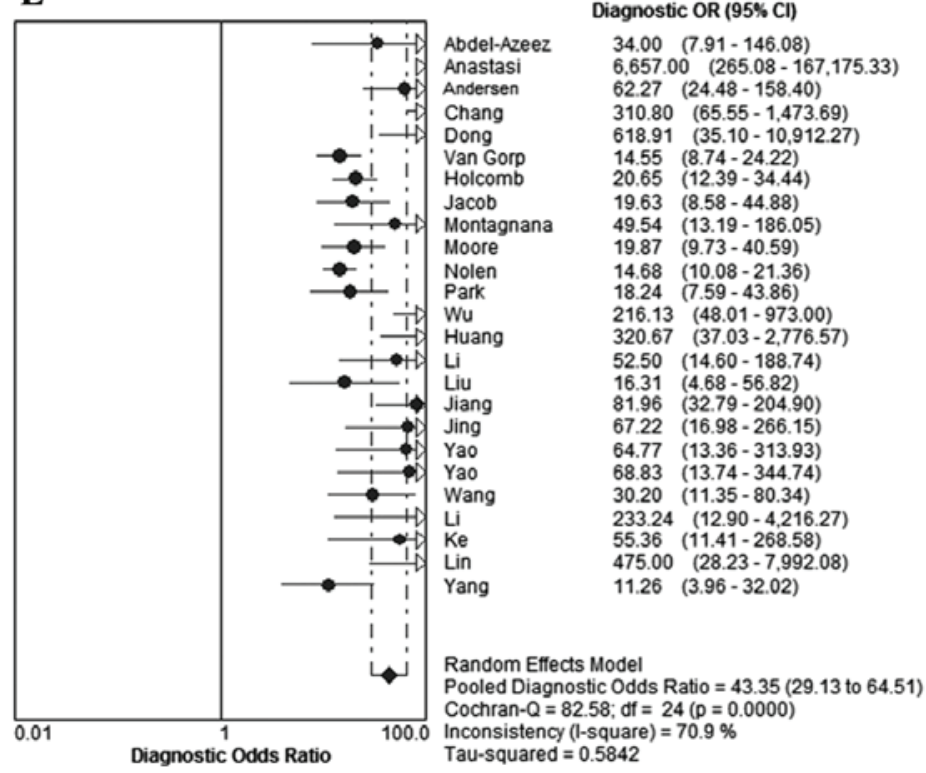

B

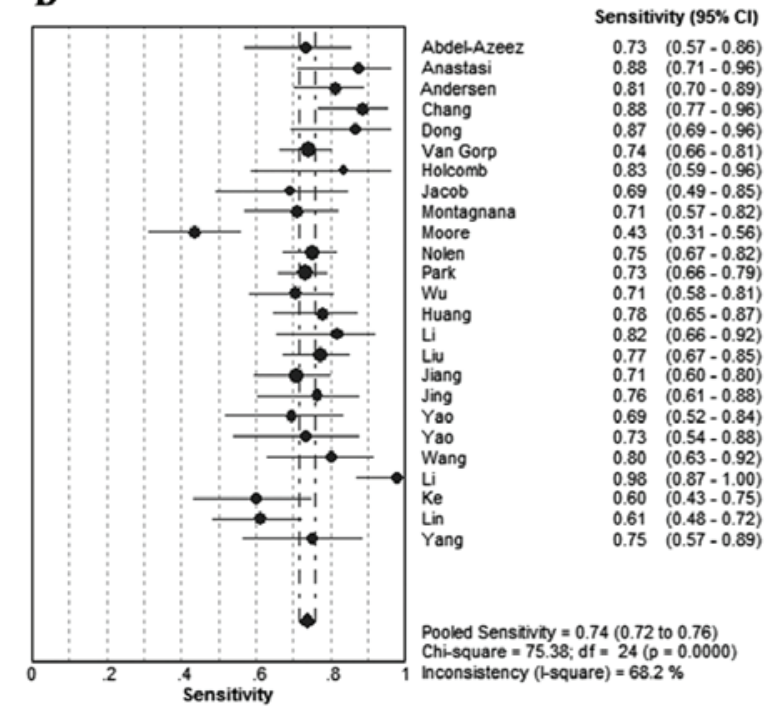

D

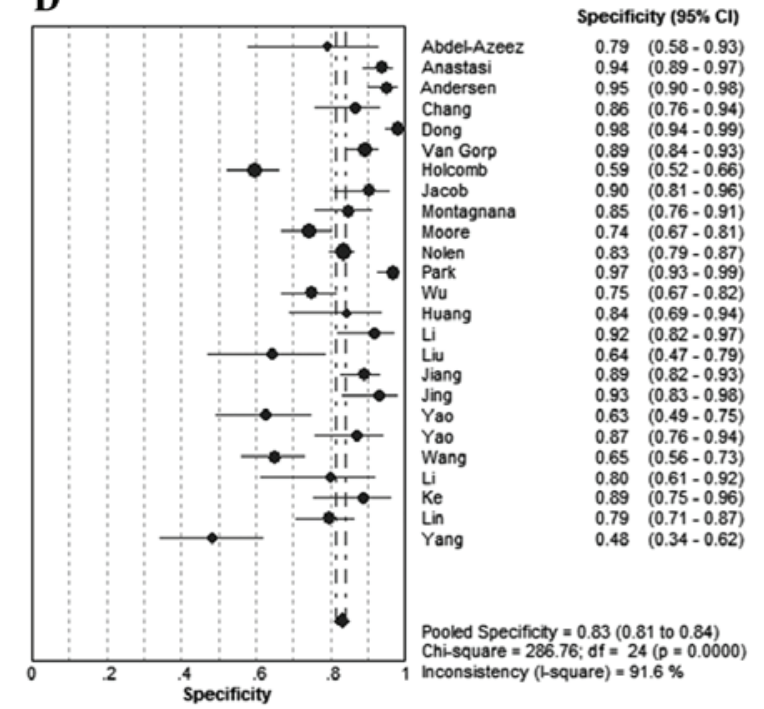

$\mathbf{F}$

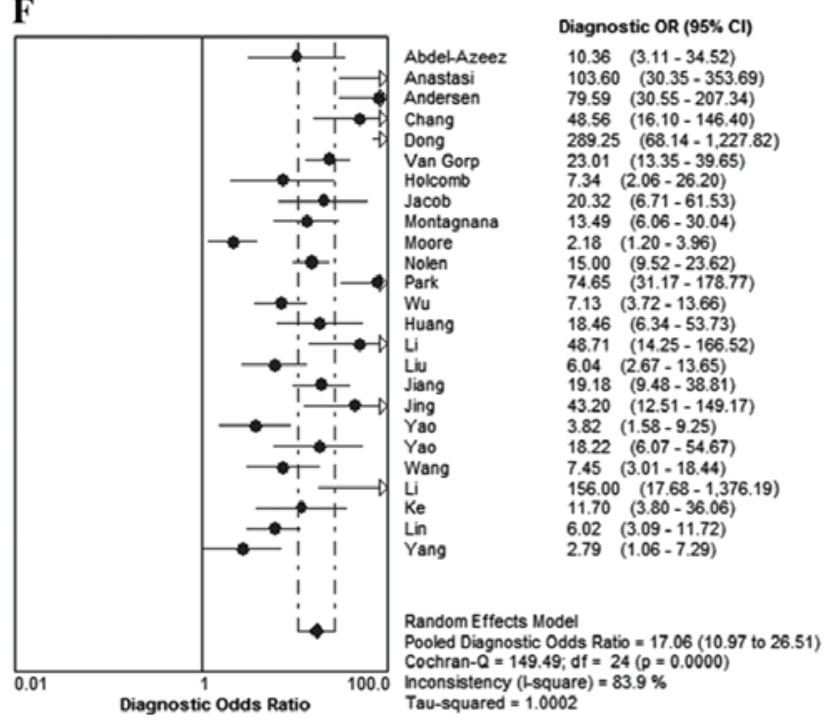

Figure 3. Sensitivity and specificity plots of (A and C) human epididymis protein 4 (HE4); and (B and D) carbohydrate antigen 125 (CA125).(E and F) Summary diagnostic odds ratio for HE4 or CA125 determination in the diagnosis of ovarian cancer. 
Table II. Characteristics of studies included in the analysis.

\begin{tabular}{|c|c|c|c|c|c|c|c|}
\hline Author (year) & Location & No. & Cut-off value & Test method & $\begin{array}{l}\text { Study } \\
\text { design }\end{array}$ & $\begin{array}{l}\text { Patient } \\
\text { enrollment }\end{array}$ & (Refs.) \\
\hline Abdel-Azeez et al (2010) & Egypt & 65 & HE4: 72 pmol/l; CA125: $35 \mathrm{U} / \mathrm{ml}$ & HE4: ELISA; CA125: ELISA & ND & ND & (22) \\
\hline Anastasi et al (2010) & Italy & 190 & HE4: $150 \mathrm{pmol} / \mathrm{l}$; CA125: $35 \mathrm{U} / \mathrm{ml}$ & HE4: ELISA; CA125: RIA & $\mathrm{P}$ & $\mathrm{C}$ & (23) \\
\hline Andersen et al (2010) & USA & 211 & $\begin{array}{l}\text { HE4: upper } 95 \text { th percentile } \\
\text { of benign groups }\end{array}$ & HE4: ELISA; CA125: ELISA & $\mathrm{P}$ & $\mathrm{C}$ & (24) \\
\hline Chang et al (2011) & China & 202 & HE4: $150 \mathrm{pmol} / \mathrm{l}$; CA125: $35 \mathrm{U} / \mathrm{ml}$ & HE4: ELISA; CA125: ELISA & ND & $\mathrm{C}$ & (2) \\
\hline Van Gorp et al (2011) & Belgium & 389 & HE4: 70 pmol/1; CA125: $35 \mathrm{U} / \mathrm{ml}$ & HE4: ELISA; CA125: EIA & $\mathrm{P}$ & $\mathrm{C}$ & $(25)$ \\
\hline Holcomb et al (2011) & USA & 229 & HE4: 70 pmol/l; CA125: $35 \mathrm{U} / \mathrm{ml}$ & HE4: ELISA; CA125: ELISA & $\mathrm{P}$ & $\mathrm{C}$ & (26) \\
\hline Jacob et al (2011) & Switzerland & 160 & HE4: 70 pmol/l; CA125: $35 \mathrm{U} / \mathrm{ml}$ & HE4: ELISA; CA125: ELISA & $\mathrm{P}$ & ND & (27) \\
\hline Montagnana et al (2011) & Italy & 104 & HE4: $74.2 \mathrm{pmol} / \mathrm{l}$; CA125: $35 \mathrm{U} / \mathrm{ml}$ & HE4: ELISA; CA125: ELISA & $\mathrm{R}$ & $\mathrm{C}$ & (28) \\
\hline Moore et al (2008) & USA & 233 & HE4: $70 \mathrm{pmol} / 1$ & HE4: ELISA; CA125: RIA & $\mathrm{R}$ & $\mathrm{C}$ & (29) \\
\hline Nolen et al (2010) & USA & 790 & HE4: $38.5 \mathrm{pmol} / 1$; CA125: $35 \mathrm{U} / \mathrm{ml}$ & HE4: ELISA; CA125: ELISA & ND & ND & $(30)$ \\
\hline Park et al (2012) & Korea & 323 & HE4: 70 pmol/l; CA125: $35 \mathrm{U} / \mathrm{ml}$ & HE4: EIA; CA125: CLIA & $\mathrm{R}$ & $\mathrm{C}$ & $(31)$ \\
\hline Dong et al (2008) & China & 212 & HE4: 70 pmol/l; CA125: $35 \mathrm{U} / \mathrm{ml}$ & HE4: ELISA; CA125: ELISA & $\mathrm{P}$ & $\mathrm{C}$ & $(32)$ \\
\hline Wu et al (2012) & China & 203 & HE4: $150 \mathrm{pmol} / \mathrm{l}$; CA125: $35 \mathrm{U} / \mathrm{ml}$ & HE4: ELISA; CA125: ELISA & $\mathrm{P}$ & $\mathrm{C}$ & (33) \\
\hline Yang et al (2010) & China & 86 & HE4: $150 \mathrm{pmol} / \mathrm{l}$; CA125: $35 \mathrm{U} / \mathrm{ml}$ & HE4: ELISA; CA125: ELISA & $\mathrm{P}$ & $\mathrm{C}$ & (34) \\
\hline Huang and Zeng (2011) & China & 96 & HE4: $150 \mathrm{pmol} / \mathrm{l}$; CA125: $35 \mathrm{U} / \mathrm{ml}$ & HE4: ELISA; CA125: ELISA & $\mathrm{P}$ & $\mathrm{C}$ & $(35)$ \\
\hline Li et al (2013) & China & 98 & HE4: 70 pmol/1; CA125: $35 \mathrm{U} / \mathrm{ml}$ & HE4: ELISA; CA125: ELISA & $\mathrm{P}$ & $\mathrm{C}$ & $(36)$ \\
\hline Liu et al (2010) & China & 131 & HE4: 75 pmol/1; CA125: $35 \mathrm{U} / \mathrm{ml}$ & HE4: ELISA; CA125: ELISA & $\mathrm{P}$ & $\mathrm{C}$ & $(37)$ \\
\hline Jiang et al (2010) & China & 225 & HE4: 46.15 pmol/1; CA125: $35 \mathrm{U} / \mathrm{ml}$ & 1HE4: ELISA; CA125: ELISA & $\mathrm{R}$ & $\mathrm{C}$ & (38) \\
\hline Jing et al (2011) & China & 100 & HE4: $150 \mathrm{pmol} / \mathrm{l}$; CA125: $35 \mathrm{U} / \mathrm{ml}$ & HE4: ELISA; CA125: ELISA & $\mathrm{P}$ & $\mathrm{C}$ & (39) \\
\hline Yao and Hong (2012) & China & 95 & HE4: $150 \mathrm{pmol} / \mathrm{l}$; CA125: $35 \mathrm{U} / \mathrm{ml}$ & HE4: ELISA; CA125: ELISA & $\mathrm{P}$ & $\mathrm{C}$ & $(40)$ \\
\hline Yao et al (2010) & China & 91 & HE4: 70 pmol/1; CA125: $35 \mathrm{U} / \mathrm{ml}$ & HE4: EIA; CA125: ECLIA & $\mathrm{P}$ & $\mathrm{C}$ & $(41)$ \\
\hline Wang et al (2010) & China & 161 & HE4: 70 pmol/l; CA125: $35 \mathrm{U} / \mathrm{ml}$ & HE4: ELISA; CA125: ELISA & $\mathrm{P}$ & $\mathrm{C}$ & $(42)$ \\
\hline Li et al (2013) & China & 70 & HE4: 70 pmol/l; CA125: $35 \mathrm{U} / \mathrm{ml}$ & HE4: ELISA; CA125: ELISA & $\mathrm{P}$ & $\mathrm{C}$ & (43) \\
\hline Ke and Liu (2010) & China & 84 & HE4: 70 pmol/1; CA125: $35 \mathrm{U} / \mathrm{ml}$ & HE4: EIA; CA125: ELISA & $\mathrm{P}$ & $\mathrm{C}$ & (44) \\
\hline Lin et al (2013) & China & 181 & HE4: 150 pmol/l; CA125: 35 U/ml & HE4: ELISA; CA125: ELISA & $\mathrm{R}$ & $\mathrm{C}$ & $(45)$ \\
\hline
\end{tabular}

HE4, human epididymis protein 4; CA125, carbohydrate antigen 125; ELISA, enzyme-linked immunosorbent assay; RIA, radioimmunoassay; EIA, enzyme immunoassay; CLIA, chemiluminescence immunoassay; ECLIA, electrochemiluminescence assay; P, prospective; C, consecutive; R, retrospective;ND, not defined.

Quality assessment of the included studies. The methodological quality assessment for the included studies is shown in Fig. 2. All the studies included in our meta-analysis met on average 10 of the 14 QUADAS criteria, reflecting high quality.

Data synthesis and meta-analysis. The results revealed that the pooled sensitivities and 95\% CIs for HE4 and CA125 were $0.74(0.72-0.76)$ and $0.74(0.72-0.76)$, respectively. The pooled specificities and respective 95\% CIs for HE4 and CA125 were $0.90(0.89-0.91)$ and $0.83(0.81-0.84)$, respectively. The summary DORs and $95 \%$ CIs for HE4 and CA125 were 43.35 (29.13-64.51) and 17.06 (10.97-26.51), respectively (Fig. 3). The SROC curve, which illustrates the correlation between sensitivity and specificity, was obtained using the random effects model to present the overall summary of HE4 and CA125. The PLRs and respective 95\% CIs for HE4 and CA125 were 10.59 (7.20-15.58) and 4.84 (3.59-6.54), respectively. The NLRs and $95 \%$ CIs for HE4 and CA125 were 0.27 (0.24-0.31) and 0.31 (0.26-0.38), respectively. The AUC for HE4 and CA125 was 0.8915 and 0.8538 , respectively. These findings indicated that HE4 and CA125 may be useful biomarkers for OC diagnosis and HE4 appears to be superior to CA125 regarding

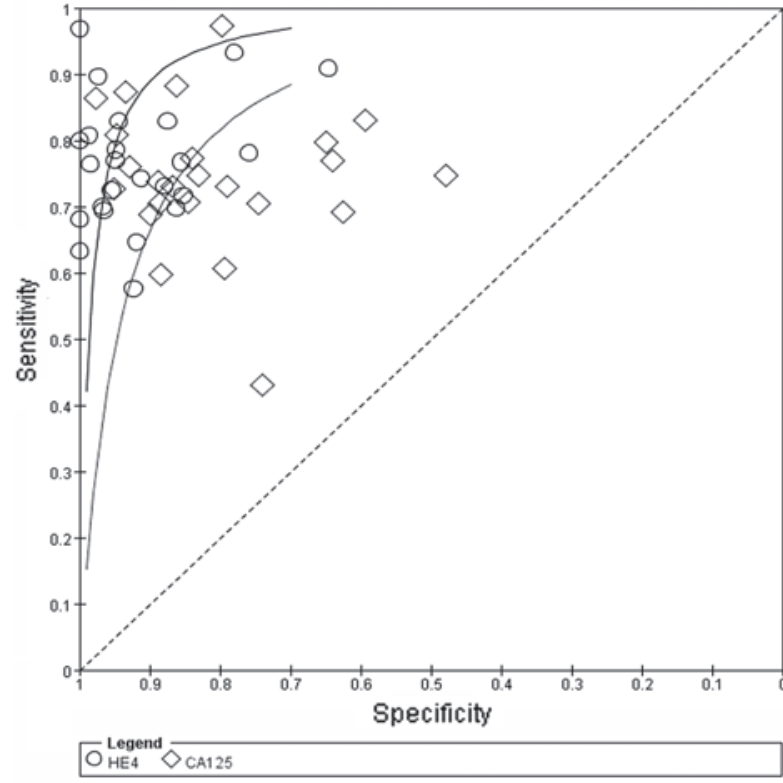

Figure 4. Summary receiver operating characteristic curves from the linear regression analysis for human epididymis protein 4 (HE4) and carbohydrate antigen 125 (CA125). 
$\mathbf{A}$

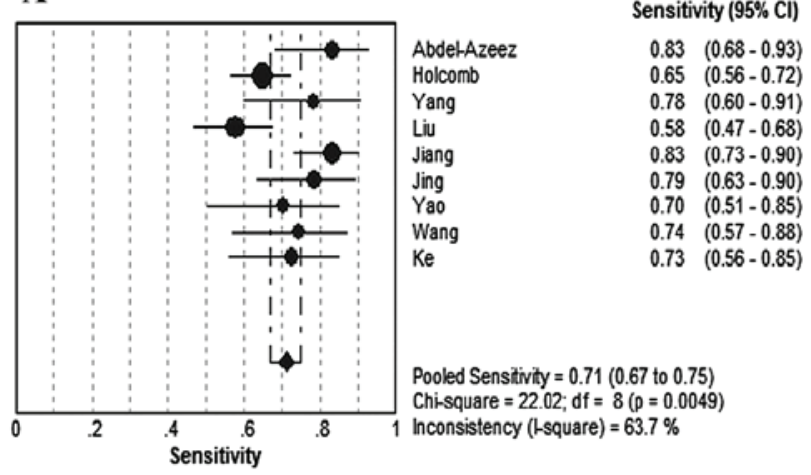

C

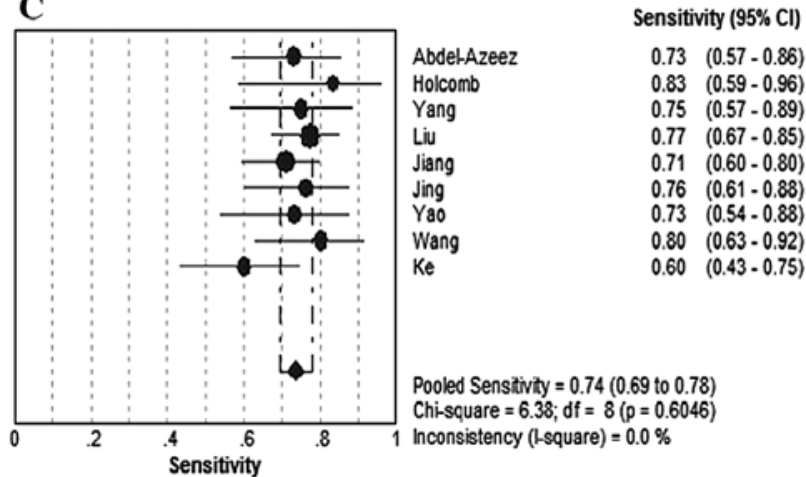

E

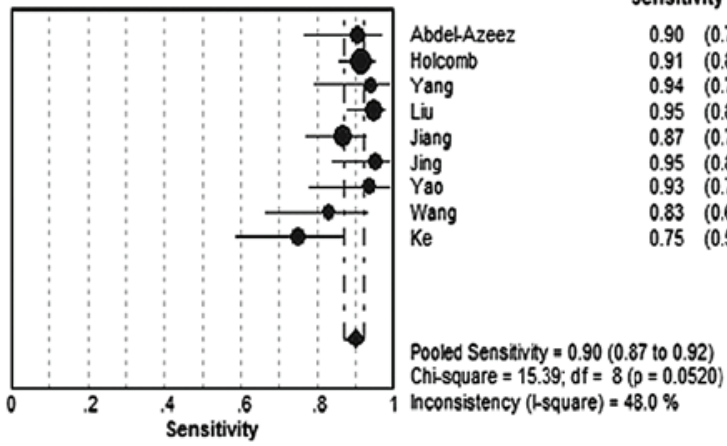

B

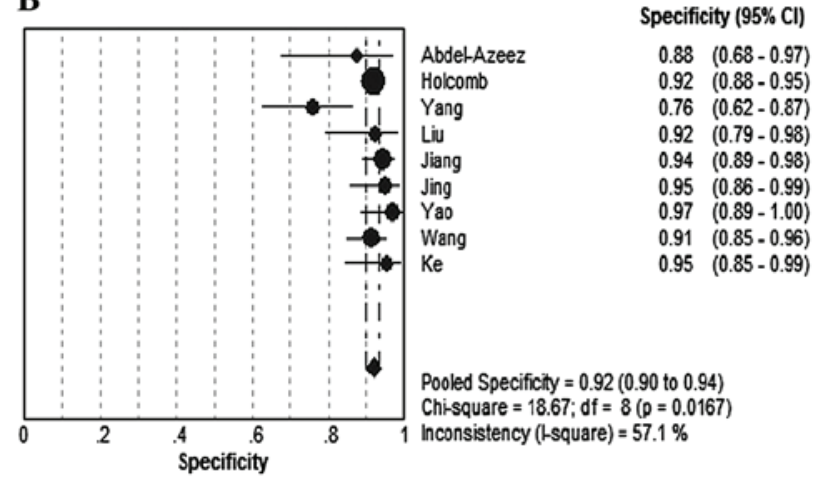

D

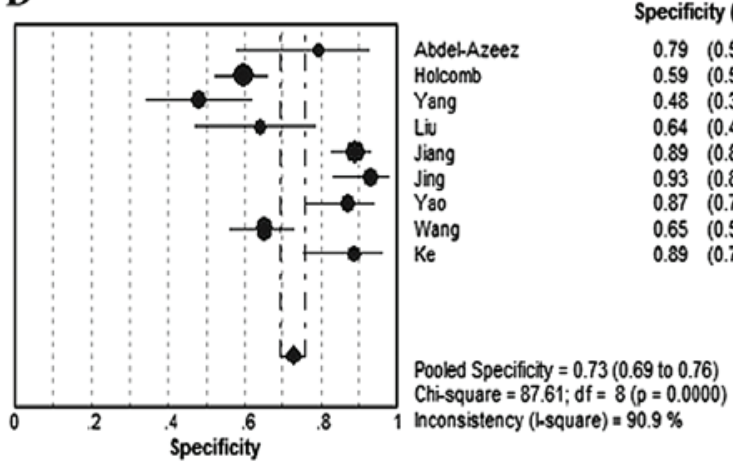

$\mathbf{F}$

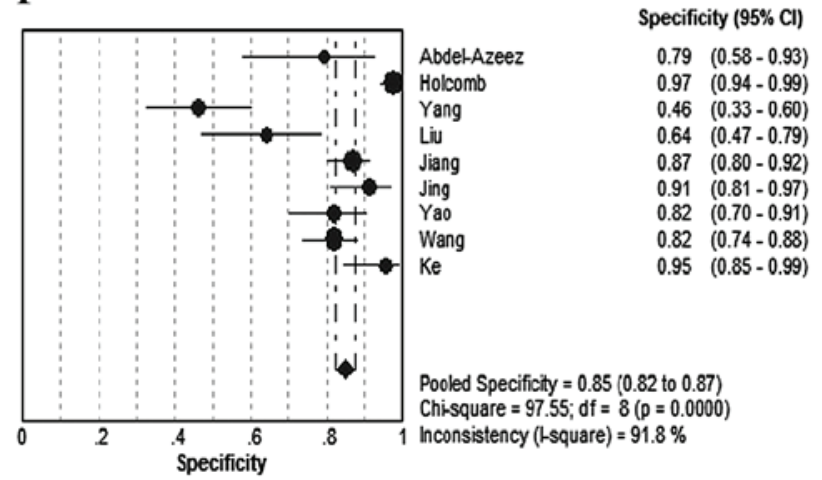

Figure 5. A subgroup of nine studies. Sensitivity and specificity plots, respectively, of (A and B) human epididymis protein 4 (HE4); (C and D) carbohydrate antigen 125 (CA125); and (E and F) HE4+CA125.

diagnostic accuracy in distinguishing $\mathrm{OC}$ from other benign gynecological diseases (Fig. 4).

In total, 9 studies investigated the diagnostic accuracy of HE4 combined with CA125 for the diagnosis of OC. The data are presented in Fig. 5. The pooled sensitivity and 95\% CIs for HE4, CA125 and HE4+CA125 in this subgroup were 0.71 (0.67-0.75), 0.74 (0.69-0.78) and 0.90 (0.87-0.92), respectively; the pooled specificity and 95\% CIs for HE4, CA125 and HE4+CA125 were 0.92 (0.90-0.94), 0.73 (0.69-0.76) and 0.85 (0.82-0.87), respectively. In this subgroup, the sensitivity of HE4 combined with CA125 was significantly elevated compared to that of HE4 or CA125 alone (Fig. 6). The pooled DORs and 95\% CIs for HE4, CA125 and HE4+CA125 were 31.83 (19.77-51.26), 10.31 (6.18-17.21) and 53.92 (26.07-111.54), respectively.
Publication bias and heterogeneity assessment. The asymmetry of the funnel plots using Egger's and Begg's tests revealed that there was publication bias among the included studies (Fig. 7). In addition, the heterogeneity was significant among the included studies. A random effects model was used and meta-regression was used to explain the heterogeneity by investigating the study characteristics; however, we observed that the differences in race, cut-off value and study design did not exert a statistically significant effect on diagnostic accuracy. In total, 22 studies were filtered (all using ELISA for the HE4 test) and the $\mathrm{I}^{2}$ of sensitivity for HE4 in these 22 studies was low; however, the $\mathrm{I}^{2}$ of specificity was not as low as anticipated. Further studies are required to confirm the role of cut-off value and race in the diagnostic accuracy for OC. 


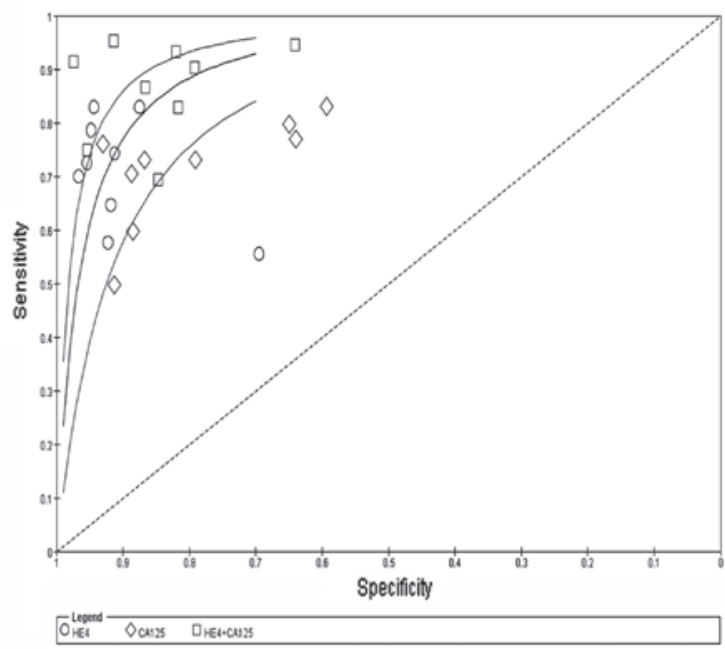

Figure 6. Summary receiver operating characteristic curves for human epididymis protein 4 (HE4), carbohydrate antigen 125 (CA125) and HE4+CA125.

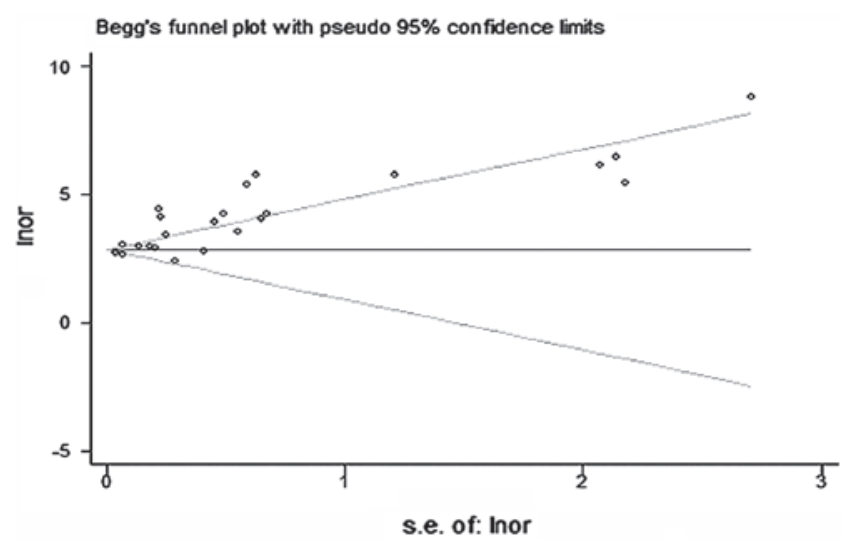

Figure 7. Funnel plot of the included studies.

The asymmetry of the funnel plots using Egger's and Begg's tests demonstrated that there was publication bias among the included studies.

\section{Discussion}

Due to the lack of sensitive and specific screening methods, $\mathrm{OC}$ is the leading cause of gynecological malignancy-related mortality in the USA and Western Europe. The main aim for laboratory biomarkers is to accurately detect $\mathrm{OC}$ at an early stage (46). Compared to transvaginal ultrasound, OC serum markers are more convenient and cost-effective. HE4, as a novel serum biomarker for $\mathrm{OC}$, has been reported as the most promising assistant marker in OC diagnosis. The WFDC2 gene, which encodes the HE4 protein, was confirmed as being overexpressed in OC but not in normal tissue (7). HE4 has been suggested to have a diagnostic sensitivity similar to that of CA125 and an increased diagnostic specificity in patients with gynecological malignancies (47).

However, the few available meta-analyses evaluating these diagnostic values are affected by several limitations. In the study by Yu et al (48), a healthy population was enrolled as the control group, resulting in a possible spurious increase in the efficacy of the biomarker compared to that from a clinically relevant population. Thus, the evaluation of the diagnostic performance of the combined measurements of HE4 and CA125 was not considered.

In this study, we assessed the included studies that compared HE4 with CA125 in the same population. Our results demonstrated that women with gynecological disease and increased concentrations of HE4 or CA125 exhibit a higher risk of malignancy. The summary DOR of HE4 was higher compared to that of CA125 (29.07 vs. 20.99). In particular, the sensitivity of HE4 was higher compared to that of CA125 (0.74 vs. 0.73), whereas HE4 exhibited a higher specificity compared to that of CA125 (0.87 vs. 0.84). The LR calculation confirmed that HE4 outperforms CA125 in identifying OC (LR+, 6.92 vs. 5.75), whereas the ability to rule out $\mathrm{OC}$ was quite similar for the two markers and rather poor. A subgroup was established to assess the two factors and we observed that the sensitivities of the subgroup exhibited significant homogeneity. These results further support the hypothesis that HE4 and CA125 may be useful biomarkers for OC diagnosis and HE4 may replace CA125 as a standalone biochemical test for OC diagnosis. Futhermore, an increase in sensitivity was achieved by combining HE4 with CA125. A meta-regression identified cut-off value and race as the major sources of heterogeneity. CA125 was the only U.S. Food and Drug Administration (FDA)-approved biomarker for OC prior to 2008 and HE4 was approved as a marker of epithelial OC by the FDA in 2008; therefore, more robust estimates of the diagnostic performance of HE4 are required.

There were certain limitations to our meta-analysis. First, there are $>4,000$ studies on the application of CA125 as a diagnostic tool for OC, whereas only $~ 50$ studies on HE4 have been published in PubMed, due to its recent identification during genomic research. Consequently, there are not enough studies to accurately evaluate the performance of HE4 in this clinical setting. Second, our study evaluated the performance of HE4 regardless of the menopausal status, as menopausal status is not marginal, since higher HE4 concentrations are detectable in postmenopausal women. Therefore, HE4 may be different, as for OC histological subtypes and OC International Federation of Gynecology and Obstetrics stages. Third, the results present with a significant publication bias for HE4 studies and heterogeneity among retrieved studies. Finally, the definition of specific clinical thresholds may be required for pre- and postmenopausal women.

In conclusion, this meta-analysis provided encouraging preliminary evidence that the measurement of HE4 may be superior to CA125 regarding diagnostic performance in OC. Furthermore, a combination of HE4 and CA125 may achieve increased diagnostic sensitivity and specificity; however, heterogeneity requires further investigation. Large-scale long-term studies should be performed to determine the clinical use of HE4 and CA125 as tumor markers for OC.

\section{Acknowledgements}

This study was supported by grants from the National Natural Science Foundation of China (grant no. 81101957). 


\section{References}

1. Siegel R, Ward E, Brawley O and Jemal A: Cancer statistics, 2011: the impact of eliminating socioeconomic and racial disparities on premature cancer deaths. CA Cancer J Clin 61: 212-236, 2011

2. Chang X, Ye X, Dong L, et al: Human epididymis protein 4 (HE4) as a serum tumor biomarker in patients with ovarian carcinoma. Int J Gynecol Cancer 21: 852-858, 2011.

3. Fountain J, Trimble E and Birrer MJ: Summary and discussion of session recommendations. Gynecol Oncol 103: S23-S25, 2006.

4. Buamah P: Benign conditions associated with raised serum CA-125 concentration. J Surg Oncol 75: 264-265, 2000.

5. Yurkovetsky Z, Skates S, Lomakin A, et al: Development of a multimarker assay for early detection of ovarian cancer. J Clin Oncol 28: 2159-2166, 2010.

6. Cree IA: Improved blood tests for cancer screening: general or specific? BMC Cancer 11: 499, 2011

7. Drapkin R, von Horsten HH, Lin Y, et al: Human epididymis protein 4 (HE4) is a secreted glycoprotein that is overexpressed by serous and endometrioid ovarian carcinomas. Cancer Res 65: $2162-2169,2005$

8. Galgano MT, Hampton GM and Frierson HF Jr: Comprehensive analysis of HE4 expression in normal and malignant human tissues. Mod Pathol 19: 847-853, 2006.

9. Partheen K, Kristjansdottir B and Sundfeldt K: Evaluation of ovarian cancer biomarkers HE4 and CA-125 in women presenting with a suspicious cystic ovarian mass. J Gynecol Oncol 22: 244-252, 2011

10. Li F, Tie R, Chang K, et al: Does risk for ovarian malignancy algorithm excel human epididymis protein 4 and CA125 in predicting epithelial ovarian cancer: a meta-analysis. BMC Cancer 12: 258, 2012.

11. Moher D, Liberati A, Tetzlaff J and Altman DG; PRISMA Group: Preferred reporting items for systematic reviews and meta-analyses: the PRISMA statement. J Clin Epidemiol 62: 1006-10012, 2009.

12. Stroup DF, Berlin JA, Morton SC, et al: Meta-analysis of observational studies in epidemiology: a proposal for reporting. Meta-analysis Of Observational Studies in Epidemiology (MOOSE) group. JAMA 283: 2008-2012, 2000

13. Whiting PF, Rutjes AW, Westwood ME, et al: QUADAS-2: a revised tool for the quality assessment of diagnostic accuracy studies. Ann Intern Med 155: 529-536, 2011.

14. Bossuyt PM, Reitsma JB, Bruns DE, et al; Standards for Reporting of Diagnostic Accuracy: Towards complete and accurate reporting of studies of diagnostic accuracy: the STARD initiative. Standards for Reporting of Diagnostic Accuracy. Clin Chem 49: 1-6, 2003

15. Devillé WL, Buntinx F, Bouter LM, et al: Conducting systematic reviews of diagnostic studies: didactic guidelines. BMC Med Res Methodol 2: 9, 2002.

16. DerSimonian R and Laird N: Meta-analysis in clinical trials. Control Clin Trials 7: 177-188, 1986

17. Pepe MS, Feng Z, Janes H, et al: Pivotal evaluation of the accuracy of a biomarker used for classification or prediction: standards for study design. J Natl Cancer Inst 100: 1432-1438, 2008

18. Glas AS, Lijmer JG, Prins MH, et al: The diagnostic odds ratio: a single indicator of test performance. J Clin Epidemiol 56: 1129-1135, 2003

19. Walter SD: Properties of the summary receiver operating characteristic (SROC) curve for diagnostic test data. Stat Med 21: $1237-1256,2002$

20. Huedo-Medina TB, Sánchez-Meca J, Marín-Martínez F and Botella J: Assessing heterogeneity in meta-analysis: Q statistic or $I^{2}$ index? Psychol Methods 11: 193-206, 2006.

21. Dinnes J, Deeks J, Kirby J and Roderick P: A methodological review of how heterogeneity has been examined in systematic reviews of diagnostic test accuracy. Health Technol Assess 9: $1-113,2005$.

22. Abdel-Azeez HA, Labib HA, Sharaf SM and Refai AN: HE4 and mesothelin: novel biomarkers of ovarian carcinoma in patients with pelvic masses. Asian Pac J Cancer Prev 11: 111-116, 2010.

23. Anastasi E, Marchei GG, Viggiani V, et al: HE4: a new potential early biomarker for the recurrence of ovarian cancer. Tumor Biol 31: 113-119, 2010.

24. Andersen MR, Goff BA, Lowe KA, et al: Use of a Symptom Index, CA125, and HE4 to predict ovarian cancer. Gynecol Oncol 116: 378-383, 2010.
25. Van Gorp T, Cadron I, Despierre E, et al: HE4 and CA125 as a diagnostic test in ovarian cancer: prospective validation of the Risk of Ovarian Malignancy Algorithm. Br J Cancer 104: 863-870, 2011

26. Holcomb K, Vucetic Z, Miller MC and Knapp RC: Human epididymis protein 4 offers superior specificity in the differentiation of benign and malignant adnexal masses in premenopausal women Am J Obstet Gynecol 205: 358.e1-358.e6, 2011.

27. Jacob F, Meier M, Caduff R, et al: No benefit from combining HE4 and CA125 as ovarian tumor markers in a clinical setting. Gynecol Oncol 121: 487-491, 2011.

28. Montagnana M, Danese E, Ruzzenente O, et al: The ROMA (Risk of Ovarian Malignancy Algorithm) for estimating the risk of epithelial ovarian cancer in women presenting with pelvic mass: is it really useful? Clin Chem Lab Med 49: 521-525, 2011.

29. Moore RG, Brown AK, Miller MC, et al: The use of multiple novel tumor biomarkers for the detection of ovarian carcinoma in patients with a pelvic mass. Gynecol Oncol 108: 402-408, 2008.

30. Nolen B, Velikokhatnaya L, Marrangoni A, et al: Serum biomarker panels for the discrimination of benign from malignant cases in patients with an adnexal mass. Gynecol Oncol 117: 440-445, 2010.

31. Park Y, Kim Y, Lee EY, et al: Reference ranges for HE4 and CA125 in a large Asian population by automated assays and diagnostic performances for ovarian cancer. Int J Cancer 130: 1136-1144, 2012.

32. Dong L, Cheng XH, Ye X, et al: The values of serum human epididymis secretory protein 4 and $\mathrm{CA}(125)$ assay in the diagnosis of ovarian malignancy. Chin J Obstet Gynecol 43: 931-936, 2008 (In Chinese).

33. Wu XW, Fu GY, Wang R and Shi XQ: Significance of using combined assays of serum human epididymis secretory protein 4 , CA125 and ROMA in the diagnosis of ovarian malignancy and pelvic mass. J Basic Clin Oncol 5: 25, 2012

34. Yang $\mathrm{C}$, Song ML, Zhong HB, et al: The differential diagnostic value of HE4, CA125 and the risk of ovarian malignancy aligorithm in ovarian tumor. Suzhou Univ J Med Sci 30: 4, 2010.

35. Huang $S$ and Zeng Q: The clinical value of HE4 and CA125 combined with sB7-H4 in early diagnosis of ovarian cancer. Hebei Med 17: 443, 2011 (In Chinese).

36. Li Q, Song X, Wu Q, et al: Clinical application of combined HE4 and CA125 differentiate malignant ovarian tumors from ovarian endometriotic cysts. Mod Oncol 21: 2, 2013.

37. Liu G, Wang A, Liu Q, et al: Combined detection of serum CA125 and human epididymis protein 4 levels in ovarian cancer. Chin J Clin Lab Sci 28: 119-121, 2010 (In Chinese).

38. Jiang DL, Sun JM, Cai LL, et al: Changes and clinical significance of serum HE4 in patients with ovarian cancer. J Pract Med 26: 14, 2010.

39. Jing XG, Wang GJ, Pei YX, et al: Diagnostic value of combined measurement of CA125, HE4 and imaging examination patients with epithelial ovarian cancer. J Third Mil Med Univ 33: 6, 2011.

40. Yao YX and Hong W: Clinical significance of detecting serum HE4, CA125 and CA724 levels in diagnoses of ovarian malignancies. Labeled Immunoassays Clin Med 19: 3, 2012 (In Chinese).

41. Yao YL, Liu Q and Li XY: The diagnostic values of combined determination of serum tumor markers HE4, TPS and CA125 levels in patients with ovarian cancer. J Radioimmunol 23: 4 , 2010 (In Chinese).

42. Wang KY, Leng JH, Zheng H and Jiang LH: Studies on value of combination of human epididymis protein 4 and CA125 in patients with ovarian cancer. Chin J Health Lab Technol 20: 1139-1140, 2010.

43. Li ZJ, Zheng YQ and Xu XF: Clinic value of HE4, CA125 combined with risk of ovarian malignancy algorithm (ROMA) in the diagnosis for ovarian cancer. J Chin Oncol 19: 219-222, 2013.

44. Ke and Liu F: Serum HE4 and CA125 in the diagnosis of ovarian cancer. Mod Hosp 10: 5, 2010.

45. Lin YY, Chen Y, Hu MH, et al: Significance of HE4 detection for diagnosis of ovarian cancer as compared with CA125 in 69 cases. Curr Immun 33: 1, 2013.

46. Moore RG and Bast RC Jr: How do you distinguish a malignant pelvic mass from a benign pelvic mass? Imaging, biomarkers, or none of the above. J Clin Oncol 25: 4159-4161, 2007.

47. Lin J, Qin J and Sangvatanakul V: Human epididymis protein 4 for differential diagnosis between benign gynecologic disease and ovarian cancer: a systematic review and meta-analysis. Eur J Obstet Gynecol Reprod Biol 167: 81-85, 2013.

48. Yu S, Yang HJ, Xie SQ and Bao YX: Diagnostic value of HE4 for ovarian cancer: a meta-analysis. Clin Chem Lab Med 50: $1439-1446,2012$. 